\title{
LENGUA NATIVA Y LENGUA DE ACOGIDA. ANÁLISIS DEL DISCURSO DEL ALUMNADO MAGREBÍ
}

\author{
BÁRBARA HERRERO MUÑOZ-COBO \\ Universidad de Almería \\ Grupo de Investigación Ilse
}

\section{Preliminares}

0.1. El objetivo de este artículo es describir el entramado psicolingüístico de los alumnos de origen magrebí residentes en España, urdido fundamentalmente por dos lenguas: el español, lengua de acogida, y el árabe marroquí ${ }^{1}$, su lengua de partida, para observar en el aula sus percepciones, trazos de una identidad en construcción. En este trabajo hemos empleado la expresión lengua de partida como sinónimo de lengua materna o lengua nativa. Nos hemos centrado en la descripción de las actitudes lingüísticas de la segunda generación, la generación bisagra, en la que es todavía posible revertir la inercia hacia la pérdida progresiva de la lengua de origen que supondría, como es sabido, el primer paso hacia una aculturación, a menudo de indeseables consecuencias. Nuestra propuesta parte de la idea de complementariedad de la lengua materna y la de acogida, para avanzar hacia un bilingüismo no sustractivo que, integrando los dos modelos lingüísticos, armonice los dos referentes simbólicos. La alternancia de códigos es la estrategia lingüística que con mayor rendimiento funcional refleja, como veremos, la complementariedad de ambos paradigmas.

Este trabajo, que se centra en una realidad sociolingüística relativamente reciente, resultado del contacto lingüístico en una sociedad multicultural, es un estudio de sociolingüística infantil y juvenil basado en el habla de un colectivo escasamente estudiado como es la comunidad lingüística trasnacional. Este grupo de habla presenta dos aspectos de interés, pues parte

\footnotetext{
${ }^{1} \mathrm{El}$ árabe marroquí, también denominado dāriŷa es la variedad oral del árabe que se emplea en Marruecos, vehículo comunicativo intergeneracional y transnacional en el que los niños se comunican con su familia, con sus parientes que viven en Europa y con los que viven en Marruecos. Como ocurre en toda situación diglósica, la lengua hablada, el marroquí, se reparte el abanico funcional con otra variedad lingüística, el árabe clásico, la lengua escrita común a los países árabes.
} 
de una visión más aséptica, no influida por los distintos debates que sobre la lengua oral y la identidad están teniendo lugar en el país de origen, Marruecos además posee, como ocurre con cualquier colectivo migrante, una conciencia lingüística muy sensible.

Para ello hemos partido, como no podía ser de otro modo, del enfoque teórico sociolingüístico que concibe como esferas codependientes la lengua y la identidad del grupo que en ella se expresa. Desde un punto cualitativo, nos hemos basado en un análisis de casos con conclusiones extrapolables, de una teoría que emerge de la realidad del aula considerada como observatorio sociolingüístico.

\subsection{La sociedad multicultural como reto sociolingüistico}

Las migraciones suponen un desafío en tanto configuran un contexto rico, complejo y dinámico que la sociedad de acogida ha de afrontar con una adecuada gestión de la diversidad ${ }^{2}$. De lo contrario la diferencia, que es en sí una riqueza para ambas culturas, para ambas identidades, puede convertirse en un problema. Así, el bilingüismo debe ser concebido como una herramienta para la integración de los diferentes vectores que constituyen la identidad del colectivo inmigrante y la armonización de sus dos mundos de referencia, de los cuales las lenguas son reflejo y medio de expresión.

La enseñanza de la segunda lengua debe por eso tender a dotar al alumno inmigrante de una competencia lingüística integrada encaminada a un bilingüismo equilibrado que refleje una identidad en la que no haya descapitalización de valores culturales. Así, si bien es fundamental el aprendizaje de la lengua del país de acogida no lo es menos el de la lengua del país de origen, ya que de lo contrario se corre el peligro de que las nuevas generaciones, tras un proceso de pérdida lingüística, vayan olvidando su lengua materna y acaben por no entender, no sólo a su familia de Marruecos, su familia extensa, sino incluso a sus propios padres, con la disfunción en todos los órdenes. Esto podría conllevar, como afirma Chini (2004: 113) en su análisis de la relación entre el árabe y el italiano ${ }^{3}$, a que aprender la

${ }^{2}$ Los programas de atención a la diversidad para preparar a los hijos de los trabajadores inmigrantes son ATAL (aulas temporales de adaptación lingüística) y ELCO (educación en la lengua y cultura de origen). Estos programas, como apunta Mijares (2006), cometen el error de la segregación escolar que, en ocasiones, entorpece más que favorece la integración de los nuevos alumnos.

${ }^{3}$ En este sentido, el caso italiano nos resulta muy útil pues se trata un país que nos ha precedido en la acogida de inmigrantes magrebíes y la investigación sociolingüística lleva más tiempo centrándose en esta cuestión. Además, el español y el italiano, lenguas cercanas entre 
lengua del país de acogida suponga para el niño migrante asumir todo un sistema simbólico y cultural, pero sobre todo es un proceso de resocialización, de redefinición identitaria y de búsqueda de su lugar en el mundo. La autora enumera con claridad las cuatro reacciones principales en las que el niño migrante puede situarse: la primera es la resistencia cultural, que se alimenta sobre todo de los referentes de la cultura de origen. La segunda opción, la asimilación, consiste en la aceptación sin reservas de la cultura del nuevo país y con el consiguiente rechazo de la de origen. La tercera de las opciones ante la que se encuentra el menor es la marginalidad, es decir, el sentimiento de exclusión de las dos culturas y la frustración ocasionada por el carácter siempre transitorio de su situación. La cuarta opción, la más deseable, es la basada en la doble referencia, es decir, en la construcción de una identidad binaria y dialéctica resultante de la combinación de los dos mundos culturales en la dirección de una compatibilidad y complementariedad crecientes. De hecho, autores como Bettoni y Rubino (1996: 135) introducen la generación de migración como variable sociolingüística y observan cómo el fenómeno de la alternancia de códigos se produce sobre todo en la segunda generación. En la misma línea, Favaro (2012: 256) diseña un árbol genealógico lingüístico de la familia migrante según el cual si la primera generación es monolingüe en la lengua de sus padres, en la segunda generación en la que nos estamos centrando, el hijo es bilingüe y muestra gran flexibilidad comunicativa y el hábito de la mezcla, y en la tercera generación, si no se remedia, se produce una vuelta hacia el monolingüismo, pero esta vez en la lengua del país de acogida.

El reto al que se enfrentan las sociedades multiculturales consiste, pues, en conseguir una sinergia de los dos paradigmas lingüísticos y culturales de la comunidad migrante: el de partida y el de acogida, el árabe y el español, en este caso.

\section{Metodología: El AUla COMO OBSERVATORIO SOCiOlingÜístico}

Los datos en los que se basa nuestra investigación han sido recopilados en la clase de religión islámica que empezó a impartirse en tres centros escolares de la provincia de Almería (uno en la capital y los otros dos en dos municipios diferentes del denominado poniente almeriense). La asignatura se imparte desde el curso académico 2005/2006, fundamentalmente en español, lo que la convierte en una herramienta de apoyo para el aprendizaje de la lengua de acogida y desarrolla contenidos que, a menu-

\footnotetext{
sí, son nuevas para el inmigrante magrebí, a diferencia de casos como el francés, que es la lengua de la educación en todo el Magreb.
} 
do, han sido adquiridos en el entorno familiar, en las escuelas marroquíes ${ }^{4}$, en las clases de fin de semana en las "mezquitas" ${ }^{5}$ o en las clases de lengua y cultura de origen.

Aunque la observación sobre cuestiones relativas a la relación entre lengua e identidad se remonta al año 2005 -lo que posibilita percibir claramente la curva de su evolución-, en este estudio en concreto nos hemos basado en los resultados de un cuestionario llevado a cabo durante los cursos 2013/2014 y 2014/2015.

Los alumnos y sus familias, dedicadas sobre todo al trabajo agrícola, representan el grueso de informantes, unos ciento veinte niños y niñas que cada año cursan estudios de Educación Primaria y los dos primeros cursos de Educación Secundaria Obligatoria (ESO), cuyas edades oscilan entre los seis y catorce o quince años, un $40 \%$ de los cuales tienen el beréber como lengua materna y un $60 \%$, el árabe marroquí ${ }^{6}$.

El tono al realizar la encuesta ha sido informal y ha consistido en que su maestro les ha ido planteando una serie de cuestiones de un modo colectivo y oral y anotando mentalmente los resultados para posibilitar la espontaneidad y la naturalidad de las respuestas. La observación se ha basado, además de en estas charlas semidirigidas, en las anécdotas colaterales que la convivencia en el aula ha generado.

Aunque para garantizar dicha espontaneidad desviamos el foco de atención haciendo ver que nuestro objetivo era conocer las preferencias televisivas del alumnado, en realidad las preguntas de nuestra encuesta iban encaminadas a conocer su percepción, sus actitudes, respecto a las lenguas que emplea, así como hacia sus medios de adquisición. Nuestro cuestionario se estructura, como puede observarse a continuación, en tres bloques. El primer bloque está constituido por una serie de preguntas que completan la clasificación sociolingüística del informante: su procedencia, su edad y su punto de partida al llegar a la escuela española, es decir, su nivel de escolarización en Marruecos ${ }^{7}$. El segundo bloque de preguntas ha ido

${ }^{4}$ Nos referimos aquí a los alumnos que llegan a España a través del proceso de reagrupación familiar y después de haber estado escolarizados en Marruecos, al menos hasta el tercer año de Primaria. Estos alumnos constituyen alrededor del 30\% del total de la muestra.

${ }^{5}$ El entrecomillado se debe a que aludimos a lugares de culto musulmán que no siempre responden a la representación arquitectónica clásica, sino a emplazamientos más improvisados.

${ }^{6}$ En la muestra contamos también con un porcentaje de un $3 \%$ de argelinos.

7 Analizando el perfil del alumno inmigrante marroquí escolarizado en Andalucía, Aram Hamparzoumian y Javier Barquín (2004) ponen de relieve la importancia del conocimiento previo de los alumnos que incluye su competencia en la lengua materna y el hecho de que, por el contrario, los niños magrebíes en Andalucía no son escolarizados conforme a sus conocimientos de español, a su escolarización previa en Marruecos o a la adecuación de sus conocimientos respecto al currículum andaluz. 
encaminado a vislumbrar cuáles son, en opinión de los alumnos, los medios que les han resultado más útiles en su aprendizaje del español. El tercer bloque consta de las preguntas referidas a sus actitudes hacia las lenguas que forman su universo lingüístico, cuándo, dónde y con quién las emplean, con qué función y cómo las perciben.

Las preguntas de la encuesta fueron las siguientes:

- Referidas a la procedencia:

- ¿Dónde has nacido?

- ¿De dónde eres?

- ¿Eres marroquí o español?

- ¿Y tu padre? ¿Y tu madre?

- ¿Has ido al colegio en Marruecos?

- Referidas a la adquisición lingüística:

- ¿Qué lenguas conoces?

- ¿Por qué, dónde y cómo las has aprendido?

- ¿Cuánto tiempo has tardado en aprender español?

- ¿Cuáles son, por orden de prioridad, los medios que más te han ayudado a aprenderlo? (los profesores, la televisión, los amigos, la familia)

- ¿Qué ves más, la televisión marroquí o la española?

- ¿Qué programas ves de la televisión española?

- Referidas a la conciencia y funcionalidad lingüísticas:

- ¿Cuál es tu lengua?

- ¿Cuándo la hablas?

- ¿Dónde la hablas cuando estás en España?

- ¿Con quién la hablas?

- ¿Dónde la hablas en Marruecos?

- ¿Con quién la hablas?

- ¿Cuál es tu lengua favorita?

- ¿Cuál es la lengua que más hablas?

- ¿Con quién la hablas? ¿Con tus hermanos? ¿Con tus padres? ¿Con la gente?

- ¿Qué lengua hablas cuando vas a Marruecos?

- ¿En qué lengua hablas cuando hablas solo?

- ¿En qué lengua sueñas?

\section{LAS ACTITUDES LINGÜÍSTICAS SOBRE LA ADQUISICIÓN Y FUNCIONALIDAD} DE LA LENGUA DE PARTIDA Y LA DE ACOGIDA

Los datos de dicha encuesta arrojaron los siguientes resultados: respecto a la adquisición lingüística del español son curiosamente las chicas 
las que tienen una percepción mucho más positiva de la aceleración de su aprendizaje así como de su propia autonomía y valía. La mayoría, al ser preguntadas sobre cómo han aprendido español, contestan: "yo sola, he aprendido yo solita", apostillando incluso "los maestros no valen pa ná", mientras que los chicos siempre aluden a una fuente exógena. Tanto los niños como las niñas perciben en su mayoría que las clases no les han servido como fuente principal de aprendizaje del español, sino el trato con los amigos españoles fuera del aula.

Otro resultado claro que arroja nuestra encuesta es que existe una relación sinérgica de mutuo reforzamiento entre la competencia bilingüe, la adquisición lingüística y su sensación de avance ya que, en el caso de los niños que llegaron a España con un conocimiento de dos lenguas, el árabe y el francés, su percepción del tiempo invertido en el aprendizaje del español y el tiempo real es menor que el de los que llegaron sin escolarizar.

Debemos puntualizar, no obstante, que la conciencia lingüística de nuestros alumnos no es siempre tan clara, sino que puede resultar a menudo ambivalente e incluso contradictoria. Por ejemplo, una niña de trece años que lleva nueve años en España y llegó sin haber sido escolarizada en Marruecos afirma respecto a los medios de adquisición lingüística que le han resultado más útiles: "aprendí yo sola, soy muy cotilla, maestro, si no sé algo no paro hasta entenderlo". La misma niña afirma a continuación y con rotundidad: "Doraimon me lo ha enseñado todo", y cuando se le pide que haga un ranking de qué es lo que más le ha servido para aprender español dice que lo primero es Boing, la cadena de dibujos animados; después, el colegio, y por último, los amigos.

Respecto a la televisión como medio de adquisición lingüística del español, como vehículo informal de formación glosodidáctica también se observa, como por otra parte es habitual en este tipo de encuestas, una percepción un tanto incongruente respecto a la realidad, ya que la mayoría afirma no haber aprendido gracias a este medio y algunos, incluso, declaran no ver en absoluto la televisión española, aunque en sus críticas se haga evidente lo contrario ${ }^{8}$. Lo mismo ocurre con la televisión marroquí, que dicen no ver pero que algunos conocen a la perfección. Así, una niña de once años, cuando se le pregunta qué programas ve de la televisión española, dice que Rashid Show ${ }^{9}$.

\footnotetext{
${ }^{8}$ Los programas más vistos de la televisión española son series como Aída o La que se avecina, y los dibujos animados del Disney Channel.

${ }^{9}$ La asociación entre el programa y lo español no es de extrañar, pues el planteamiento del programa, un late night que goza de un seguimiento masivo, resulta para Marruecos muy novedoso por el lenguaje directo que refleja una realidad no maquillada, algo que los niños asocian a menudo a lo español.
} 
Los hijos de parejas mixtas parecen tener, sin embargo, una mayor inclinación a ver la televisión como medio de aprendizaje del español y mayor conciencia de ello. Así, una niña nos dice: "yo he aprendido con Clan, la televisión en español; la televisión marroquí, Manar, tala li f rasi”, literalmente, "ya me he hartado de la televisión marroquí, de programas como Manar ${ }^{10 "}$. Estos hijos de parejas mixtas, es decir, de padres marroquíes casados con español o española, aprenden el árabe también por medio de la televisión. De hecho, los casos de niños cuyos padres no les dejan ver la televisión no saben prácticamente nada de la lengua de uno de sus progenitores. Aunque los alumnos son conscientes del papel formativo de la televisión a nivel lingüístico, no sólo para el español, sino sobre todo para el árabe marroquí y, por poner un ejemplo, uno de nuestros informantes le preguntaba a otro: “¿si eres marroquí para qué vas a ver la televisión marroquí?" "11, no es el primer ni el único medio de instrucción lingüística, ya que la selección que los alumnos hacen de los medios eficaces para aprender el español está encabezada por los compañeros del colegio. En segundo lugar, sitúan las clases y los profesores y, en tercer lugar, los que sabían ya algo de español antes de llegar a nuestro país afirman que un pequeño vocabulario con imágenes español-árabe les ayudó mucho en la fase inicial. La televisión y las clases del programa ATAL se sitúan al final de la selección. La máxima de uno de nuestros alumnos así lo refleja: "Maestro, la tele para el árabe marroquí y la vida para el español”.

Así, mientras las charlas con sus padres y la televisión son los medios de aprendizaje del árabe marroquí, y la conversación con sus compañeros de clase y con sus profesores son el ámbito de aprendizaje del español, el ámbito de aprendizaje del árabe clásico es, fundamentalmente, la mezquita. Lo que sí está claro, pues, es que la mayoría de los niños perciben que el español es la lengua que aprenden y emplean en el ámbito público: la calle, el colegio y los amigos, mientras el árabe marroquí o, en su caso, el beréber, es la lengua de la familia, del ámbito privado, la lengua que se emplea con los padres y se aprende en el espacio familiar, ya sea en España o con la familia extensa en Marruecos. Excepción hecha de la comunicación con los hermanos que, como ocurre en otras situaciones análogas, a pesar de producirse en el ámbito de la intimidad, tiene lugar en la lengua del entorno social, el español en este caso. Así, como observa Chini (2004: 339) en el caso italiano, se produce también una selección lingüística determinada por la distinción de los espacios público y privado: la lengua

\footnotetext{
${ }^{10}$ Manar es un serial turco de la cadena marroquí 2M doblado al árabe marroquí que lleva emitiéndose varios años y que es archiconocido por los alumnos marroquíes.

${ }^{11}$ Para más información sobre el papel de la televisión como medio de adquisición del árabe marroquí, consúltese Herrero y El Azami (en prensa).
} 
de acogida, es la lengua del mundo social, de la televisión, la escuela y los amigos, mientras que el marroquí es la lengua familiar hablada, como decimos, en el círculo privado. De hecho, los niños nacidos en España o llegados a una edad temprana emplean la palabra marroquino ${ }^{12}$ con un sentido aglutinante de la nacionalidad, la tradición, la religión y, en definitiva, todo lo que hace referencia a su mundo familiar.

Un concepto interesante es el de lengua filial que introduce Favaro (2012) al analizar la inmigración en Italia, que coloca la lengua de los hijos, y no la materna, en el epicentro referencial. Lo mismo ocurre en nuestro país en el que, efectivamente, nuestros informantes invierten la pirámide docente y la mayoría declaran enseñar a sus padres el español, actuar como mediadores lingüísticos: "Mi madre me lo pide, le digo: dame agua y así va aprendiendo; también le digo: ¿qué son los animales? l hayawanāt". Así, como ellos mismos declaran, usan frases como arali l kuchillo, dame el cuchillo, en vez de arali l mus, para que, sobre todo ellas, sus madres, aprendan palabras nuevas en español.

Se observa, asimismo, un cambio en la percepción de su lengua, pues el $90 \%$ de nuestros informantes arabófonos afirma mayoritariamente que su lengua es el árabe marroquí, cuando hace apenas cuatro años (coincidiendo con el doblaje de series televisivas al marroquí) decían que era el árabe, el árabe clásico ${ }^{13}$. Esta percepción es en cierto modo paradójica, pues la lengua que hablan la práctica totalidad del tiempo es el español.

En el grupo de niños escolarizados por primera vez en España, el árabe marroquí o dāriŷa alude tanto a la variedad lingüística hablada, como a la lengua materna de un modo general. El caso de los niños escolarizados previamente en Marruecos es diferente pues sí que son conscientes de la situación de diglosia de su país de origen y distinguen la lengua hablada, a la que se refieren como dāriŷa, del árabe que remite a la variedad escrita.

Respecto a la selección lingüística dependiente del interlocutor, la mayoría de los alumnos habla árabe con sus padres, español con sus hermanos, y con los amigos marroquíes prefieren el español, aunque el marroquí es la segunda opción.

Bettoni y Rubino (1996) introducen una variable nueva, la lengua que emplea el bilingüe al hablar solo, al hablar consigo mismo, a la que hemos añadido otra: la selección inconsciente; es decir, nos hemos preguntado, les hemos preguntado a nuestros alumnos, en qué lengua sueñan. A este

12 U otros términos empleados como sinónimos como marroquín o magribi.

${ }^{13}$ La programación lingüística implícita que ejerce la televisión marroquí con decisiones como el doblaje de series al árabe marroquí ha duplicado la audiencia y ha promovido el ascenso del índice de prestigio de esta variedad lingüística y el hecho de que haya comenzado a copar áreas que antes pertenecían al árabe clásico. 
respecto hemos observado, en primer lugar, que en ambos casos, al hablar consigo mismos y al soñar, eligen la misma lengua, es decir, que la lengua en la que dicen soñar es la misma en la que hablan con sus juguetes ${ }^{14}$. Se da, asimismo, una distinción clara entre los nacidos en Marruecos que recurren más a la lengua de partida -al árabe marroquí, los arabófonos, y al beréber, los berberófonos-, mientras que los nacidos en España recurren más al español. La única salvedad es el interlocutor. Así, un niño nacido en España dice: "sueño en español, pero si está mi padre, pues hablo en árabe". Los nacidos en España son conscientes de su bilingüismo hasta el punto de afirmar que en sus sueños y charlas imaginarias solapan ambas lenguas y en este sentido hacen afirmaciones como: "sueño en español, me lo dice mi madre. A veces en los dos, mi madre me dijo que soñé y dije 'al ‘adáw' (qué significa auxilio) y 'socorro'”, en las que se observa una vez más cómo conciencia y competencia lingüísticas suelen ir de la mano.

\section{LENGUA DE PARTIDA Y LENGUA DE ACOGIDA: LA ALTERNANCIA DE CÓdigOS COMO ESTRATEGIA HACIA UN BILINGÜISMO COMPLEMENTARIO}

A partir de las apreciaciones metalingüísticas de los informantes que hemos ido enumerando, hemos observado el carácter múltiple de los referentes de este colectivo y la importancia de potenciar un bilingüismo no sustractivo, un bilingüismo equilibrado en el que el hablante emplee la lengua pertinente según las variables del contexto (el acto de habla, el interlocutor, el tema, etc.). Así, aunque a los alumnos extranjeros se les suele desaconsejar, cuando no prohibir, hablar en sus lenguas vernáculas entre ellos durante las clases por considerar que pudiera retrasar su aprendizaje del español, hemos comprobado que, por el contrario, en su proceso de integración lingüística, el uso de la alternancia de códigos entre las dos lenguas que forman su entramado psicolingüístico, el español y el árabe, constituye parte de su proceso de adquisición de la lengua de acogida y de su adaptación e integración en el nuevo entorno ${ }^{15}$.

Se trata de potenciar un código binario cuyo carácter mixto ya no es percibido como un hándicap en el que la alternancia del árabe con el español

${ }^{14}$ Comenzamos a preguntar de ese modo que resultó mucho más comprensible para los alumnos gracias a la puntualización de una de las niñas de nuestra muestra que, al hacerle la pregunta: “¿en qué lenguas hablas sola?”, nos contestó: “¿te refieres a en qué lengua hablo a mis juguetes?".

${ }^{15}$ Luisa Martín Rojo y Laura Mijares (2007) observan cómo una frase tan significativa como "sólo en español", que a menudo se oye en los centros educativos a la hora de corregir a los niños inmigrantes, supone un proceso de pérdida cultural al no reconocer la lengua materna del alumno como una riqueza, una base sobre la que construir. 
es considerada de modo inconsciente e implícito como una posibilidad más que amplía de la capacidad comunicativa del bilingüe, y así lo reflejan los niños cuando pronuncian frases de nuestro corpus como "lo que no sé decir en árabe, pues lo digo en español”.

Además de las razones de disponibilidad léxica, la alternancia entre sus lenguas en la clase, el ámbito en el que nos hemos centrado, se produce muy a menudo para darle mayor autoridad o fuerza a un enunciado, por ejemplo, en el caso de los imperativos como la expresión extraída de nuestro corpus jsekt-u! (;callaos!) que, pronunciada en mitad de una proposición en español, tiene un efecto mucho más autoritario que la palabra “isilencio!”. A su vez, la expresión bessah otorga mayor legitimidad a lo que se dice que a su traducción española "de verdad" y had ši muhim les resulta más seria que exclamar "es importante". La alternancia entre las dos variedades lingüísticas, el árabe marroquí y el español, sirve, pues, para modular el tono de los enunciados y para expresar sentimientos intensos como el enfado con un cambio de lengua que marque cierto énfasis. Respecto a la alternancia de códigos del docente, se suele producir, asimismo, en intentos de motivación del alumnado con palabras como Yalla (vamos); en los actos de verificación de la comprensión como ìyek? (¿no?), y en los actos de contextualización como daba (ahora).

La alternancia al marroquí se produce, asimismo, en entornos conflictivos como reforzador del grupo como inside code, como lenguaje secreto, pues un alumno nos dijo: "hablo en marroquí en el patio para que no me entiendan los matones de sexto".

A estas funciones cabe añadir la función glosodidáctica de la combinación de ambas lenguas, en dos sentidos: por una parte, como hemos visto, como medio de enseñanza del español a los padres en el seno de la familia, la llamada lengua filial; por otra, como medio de refuerzo de la lengua de acogida en el aula. Así, la introducción del dāriŷa para acompañar al español en el desarrollo de la asignatura ha permitido comprobar su efecto sinérgico de redundancia altamente pedagógico, pues se consigue un doble refuerzo, el primero a nivel de contenidos y el segundo, de carácter puramente lingüístico según el cual la clase de religión islámica, impartida con un empleo profuso de la alternancia de códigos, pasa así a convertirse en una clase de aprendizaje y/o refuerzo del español.

Además, al realizar todos los alumnos la alternancia con una misma lengua, la española -contrariamente a lo que ocurre en Marruecos, donde en la parte central y sur del país la alternancia se hace con el francés- se produce un efecto de convergencia mediante la aminoración o disminución de las diferencias diatópicas de origen ${ }^{16}$. Así, como observa El Azami

${ }^{16}$ A este respecto consúltese el trabajo de El Azami (2011) sobre la alternancia de códigos como vehículo de convergencia lingüística. 
(2011: 48-49), palabras como bandar (invernadero), corda (cuerda), pista, pepino, parada, rueda, etc. ${ }^{17}$, han pasado a constituir parte del léxico común en sustitución de las palabras habitualmente empleadas: plastic, l-hbel, piste, jyar, stasion y pnue, respectivamente. Así, enumera frases como tfešat l pnue (se me ha pinchado la rueda) que, empleada por los procedentes de la zona francófona, se transforma al cabo del tiempo, de forma imperceptible y en boca del mismo hablante, en tpintchlatli e rueda (se me ha pinchado la rueda) u otras como jesni n-calcular l problema (tengo que calcular el problema) o ma q-dar ši n-terminar el tarea (no pude terminar la tarea) o enunciados como jdi-t e-ttobus $f l$ parada (he cogido el autobús en la parada), en lugar del antiguo jdi-t $l$ kar $f$ stasion que hubiera dicho al llegar, o gad n-mši n-rmi l bašuara (voy a tirar la basura) que sustituye -según confiesa el propio informante, sorprendido por la evolución y el cambio, de los que toma conciencia en un ejercicio de reflexión metalingüística- a gad n-msi n-rmi e-zbel.

Se trata, pues, de una estrategia lingüística primordial puesta al servicio de una mayor competencia lingüística del hablante en todos los niveles (léxico, sociolingüístico y discursivo), pues además de dotarlo de una mayor disponibilidad léxica, de ser un mecanismo de cohesión y convergencia grupal, de marcar el énfasis discursivo, funciona como instrumento de apoyo, de aceleración y de consolidación de lo aprendido y, en definitiva, de adaptación al nuevo entorno. Esta alternancia de códigos lingüísticos y culturales avanza hacia su progresiva armonización y refleja y hace emerger una identidad mestiza entre los migrantes bilingües de segunda generación.

\section{CONCLUSIONES: HACIA LA INTEGRACIÓN DE MODELOS LINGÜÍSTICOS Y SOCIOCULTURALES}

Así, el universo lingüístico de los niños "marroquíes del mundo" ${ }^{18}$ o, dicho de otro modo, de los alumnos magrebíes inmigrantes, consta básicamente de dos lenguas que reflejan distintos sistemas de valores, dos facetas de su identidad. A este respecto, los datos de nuestra encuesta muestran cómo el árabe marroquí empieza a percibirse como el eje de la identidad raigal, de la identidad de partida, muy asociada a su vez al elemento reli-

${ }^{17}$ Abundan, como podemos ver, términos propios del entorno dominado por la actividad agrícola que desempeñan sus padres en los invernaderos y por la diseminación de las viviendas.

${ }^{18}$ Esta es la nueva expresión acuñada en Marruecos para referirse a la emigración económica que viene a sustituir a la expresión "nuestros trabajadores en el extranjero", que tenía un cierto matiz despectivo. 
gioso y cultural. Así, uno de los alumnos, cuando se le pregunta sobre las diferentes lenguas que emplea, es decir, el árabe y el español, afirma lo siguiente: "Quiero aprender árabe porque soy árabe, soy musulmán. Mi lengua es el dāriŷa. Somos musulmanes porque somos marroquíes, no porque no comemos jamón, no porque sólo comemos cerdo halal"19. Sin embargo, tras analizar las respuestas de nuestros informantes queda clara una idea vertebradora: la identidad del colectivo compuesto por niños magrebíes inmigrantes de segunda generación en España hoy es de un modo muy palpable un elemento dinámico y complejo, no rígido ni excluyente. De hecho, a veces cuando se les pregunta: “¿cuál es tu lengua?”, algunos niños contestan: "depende de quién me lo pregunte, si eres árabe, pues te contesto que el árabe y si eres español, pues te contesto que el español”. Pues, como observa Favaro (2012: 257), la familia y los sentimientos, la religión y el pasado suelen radicarse en la lengua de origen, mientras que la organización social, el trabajo, la escuela lo hacen en la lengua de llegada. De hecho, muchos de nuestros alumnos aluden a la asignatura de religión islámica como clases de lengua árabe, lo que viene a demostrar algo importante a la hora de definir la identidad: para la mayoría de ellos, lengua, nacionalidad y religión resultan coordenadas extraordinariamente próximas.

La identidad religiosa, bastante reforzada en este colectivo (no olvidemos que se trata de alumnos de religión islámica), aparece vinculada al árabe tanto en su variedad escrita como en la oral. De hecho no son pocos los que a la pregunta: “¿de dónde eres, español o marroquí?”, han contestado: "soy musulmán" ${ }^{20}$. Aunque no se trata, como decimos, de una identidad excluyente, pues, curiosamente, algunos alumnos contestan: "soy español y musulmán”, a lo que otros sin embargo apostillan: "pero si eres español, por qué no comes jamón?”, haciendo alusión a la posible incompatibilidad entre los dos referentes.

La lengua española es percibida como la lengua portadora de valores de modernidad frente al árabe, portador de valores vinculados a la tradición de las sociedades patriarcales que a veces entra, como hemos dicho, en colisión con los que transmite la lengua española. Así, una alumna afirma: "no veo la tele, es fea y haram ${ }^{21}$, hacen cosas malas, incluso en los dibujos. Dicen palabrotas y nuestra religión no nos deja”.

No obstante, el estado de transición entre paradigmas que impulsan el cambio y los que reivindican la tradición es un hecho que tiene, como no

\footnotetext{
${ }^{19}$ Con la expresión cerdo halal se refiere a un embutido de consumo lícito para los musulmanes, pues no contiene cerdo.

${ }^{20}$ Esta asociación identidad-religión se observa claramente en la comunidad lingüística ceutí, en la que los informantes afirman a menudo que "hablan musulmán" (Herrero, 2013).

${ }^{21}$ Haram se refiere a lo ilícito dentro del sistema ético islámico.
} 
podía ser de otro modo, un claro reflejo lingüístico. El árabe clásico aparece vinculado tanto al islam como a los valores que sustentan la tradición, mientras el árabe marroquí vehicula una identidad vernácula más dinámica y, sobre todo, mestiza.

La alternancia de códigos que, como hemos visto, desempeña funciones como otorgar énfasis a un determinado enunciado, ser una herramienta de cohesión grupal y convergencia lingüística, y funcionar como potente herramienta glosodidáctica, es la estrategia lingüística que más clara y eficazmente visibiliza y da voz a la hibridez identitaria de este grupo.

Diremos, pues, que los niños marroquíes que viven en España son ciudadanos con una identidad compleja en tanto que es local y trasnacional (glocal), dinámica e híbrida, para los que la tradición misma se torna un concepto flexible y poroso. Esta identidad debe construirse por inclusión y sobre la idea de complementariedad, de adaptación y de simbiosis entre los diferentes modos de ver la vida trasmitidos por sus dos lenguas: el español, la lengua adquirida y empleada sobre todo en el ámbito social, que transmite los valores de la sociedad de acogida, y el marroquí, la lengua del ámbito privado, familiar, portadora de los valores culturales y religiosos de partida.

Por todo ello, la enseñanza del español a los niños inmigrantes debe estar encaminada a superar el bilingüismo sustractivo que produce la erosión lingüística y su reflejo más amplio, la aculturación, la pérdida de vínculos con la cultura de origen, y emplear estrategias lingüísticas destinadas a construir un bilingüismo equilibrado en el que las dos lenguas, las dos identidades, se complementen de tal manera que los alumnos muestren con total naturalidad una doble lealtad. 


\section{BIBLIOGRAFÍA}

Bettoni, C. y Rubino, A. (1996): Emigrazione e comportamento linguistico. Un'indagine su dei siciliani e dei veneti in Australia, Galatina (Lecce), Congedo editore.

ChINI, M. (2004): Plurilinguismo e immigrazione in Italia. Un'indagine sociolinguistica a Pavia e Torino, Milano, Franco Angeli.

EL AzAmi, O. (2011): "El árabe marroquí en el aula: análisis de dos mecanismos de convergencia lingüística”, en B. Herrero-Muñoz Cobo, L. M. Pérez Cañada, M. Aragón Huertas y F. Moscoso García (eds.), IV Congreso Árabe marroqui: Más allá de la oralidad, Almería, Universidad de Almería, págs. 42-55.

FAvaro, G. (2012): "Parole, lingue e alfabeti nella classe multiculturale", Italiano LinguaDue, 1, págs. 251-262.

Hamparzoumian, A. y Barquín, J. (2004): "El perfil del alumno inmigrante marroquí escolarizado en Andalucía”, Aldadis.net, 3 (noviembre 2004). Disponible en: <www.aldadis.net/revista3/03/articulo02.htm> (Consulta: 15 de enero de 2015).

Herrero, B. y El Azami, O. (en prensa): "Evolución de la conciencia lingüística de los 'marroquíes del mundo'. El papel de la televisión en el paso del árabe marroquí de tabú a eje identitario", en VI Congreso de árabe marroquí: Identidad y Conciencia Lingüistica, Madrid, 16-17 de abril de 2015.

Herrero Muñoz-Cobo, B. (2013): El árabe ceutí, un código mixto como reflejo de una identidad mestiza, Ceuta, Instituto de Estudios Ceutíes.

Martín Rojo, L. y Mijares, L. (2007): "Sólo en español: una reflexión sobre monolingüe y la realidad multilingüe en los centros escolares”, Revista de Educación, 343, págs. 93-112.

MijARES, L. (2006): Aprendiendo a ser marroquies. Inmigración diversidad lingüistica y escuela, Madrid, Ediciones del Oriente y el Mediterráneo. 